\title{
FATORES ASSOCIADOS À CAPACITAÇÃO DE AGENTES COMUNITÁRIOS DE SAÚDE EM HANSENÍASE EM PALMAS, TOCANTINS, BRASIL
}

\author{
FACTORS ASSOCIATED WITH THE TRAINING OF COMMUNITY \\ HEALTH WORKERS IN LEPROSY IN PALMAS, TOCANTINS, BRAZIL
}
Mariana do Prado Borges ${ }^{1}$, Edson José de Aleluia Júnior ${ }^{1}$, Luiz Pedro Fernandes Bonfim ${ }^{1}$, Alana Lima Moreira Rodrigues ${ }^{2}$, Lorena Dias Monteiro ${ }^{1,2}$

\section{คิ ACESSO LIVRE}

Citação: Borges MP, Júnior, EJA, Bonfim LPF, Rodrigues ALM, Monteiro LD. (2021) Fatores associados à capacitação de agentes comunitários de saúde em hanseníase em palmas, Tocantins, Brasil. Revista de Patologia do Tocantins, 8(1).

Instituição: ${ }^{1}$ Instituto Tocantinense Presidente Antônio Carlos. Faculdade de Medicina. Palmas, TO (ITPAC/PALMAS). ${ }^{2}$ Fundação Escola de Saúde Pública de Palmas, Tocantins, Brasil (FESP/PALMAS).

Autor correspondente: Lorena Dias Monteiro. Fundação Escola de Saúde Pública de Palmas. lorenamonteiro3@hotmail.com. Quadra 504 Sul, alameda 12, lote 18B. CEP.: 77021682. Palmas, Tocantins, Brasil.

Editor: Carvalho A. A. B. Medicina, Universidade Federal do Tocantins, Brasil.

Publicado: 12 de maio de 2021.

Direitos Autorais: (c) 2021 Borges et al. Este é um artigo de acesso aberto que permite o uso, a distribuição e a reprodução sem restrições em qualquer meio, desde que o autor original e a fonte sejam creditados.

Conflito de interesses: os autores declararam que não existem conflitos de interesses.

\begin{abstract}
RESUMO
Objetivo: Descrever os fatores associados à capacitação de ACS para o acompanhamento de casos de hanseníase em Palmas, Tocantins, Brasil. Métodos: Trata-se de estudo transversal, realizado com a aplicação de questionário em uma população de 301 agentes comunitários de saúde (ACS). Resultados: Um total de $48,84 \%$ dos ACS não reconheceu a perda da sensibilidade como sinal e/ou sintoma da hanseníase. A perda de pelos nas sobrancelhas e cílios não foi considerada como sinal e/ou sintoma da hanseníase por $74,42 \%$ dos ACS e mais de $50 \%$ não souberam identificar o agente causador da doença. Os fatores associados à melhor capacitação dos ACS para acompanharem os casos de hanseníase foram: ACS com mais idade (RP:1,60; IC 1,25-2,05), mulheres (RP: 1,38; IC 1,09-1,75), sentimento de confiança para acompanhar os casos (RP: 1,76; IC 1,47-2,10), ACS com duas ou mais visitas domiciliares para casos de hanseníase (RP: 1, 34; IC 1,09-1,63), ASC que não segrega pacientes na restrição de objetos de uso pessoal (RP: 1,82; IC 1,37-2,42), ASC que teve alguém doente na família (RP: 1,05; IC 1,05-1,41). Na regressão logística, o único determinante isolado para capacitação do ACS sobre hanseníase foi não orientar a separação de objetos de uso pessoal no domicílio. Conclusão: Este estudo trouxe novas evidências e poderá subsidiar estratégias destinadas ao aperfeiçoamento das atividades de controle da hanseníase.
\end{abstract}

Palavras-Chave: Hanseníase; Capacitação em Serviço; Agente Comunitário de Saúde.

\begin{abstract}
Objective: To describe the factors associated with the training of community health workers (CHW) to monitor leprosy cases in Palmas, Tocantins, Brazil. Methods: This is a cross-sectional study, carried out using a questionnaire in a population of 301 CHW. Results: A total of $48.84 \%$ of $\mathrm{CHW}$ did not recognize the loss of sensation as a sign and / or symptom of leprosy. The loss of hair on the eyebrows and eyelashes was not considered as a sign and / or symptom of leprosy by $74.42 \%$ of CHW and more than $50 \%$ did not know how to identify the causative agent of the disease. The factors associated with the better training of $\mathrm{CHW}$ to monitor leprosy cases were: CHW with older age (PR: 1.60; $\mathrm{Cl} 1.25-2.05)$, women (PR: 1.38; Cl 1.09- 1.75), feeling safe to follow up the cases (PR: 1.76; $\mathrm{Cl} 1.47-2.10), \mathrm{CHW}$ with two or more home visits for leprosy cases (PR: 1,$34 ; \mathrm{Cl} 1,09-1.63$ ), $\mathrm{CHW}$ that does not segregate patients in the restriction of objects of personal use (PR: 1.82; Cl 1.37-2.42), CHW who had someone sick in the family (PR: 1.05; IC 1.05-1.41). In logistic regression, the only isolated determinant for the $\mathrm{CHW}$ training on leprosy was not to guide the separation of objects of personal use at home. Conclusion: This study brought new evidence and may support strategies aimed at improving leprosy control activities. Keywords: Leprosy; Community Health Workers; In-service Training.
\end{abstract}




\section{INTRODUÇÃO}

A hanseníase, causada por Mycobacterium leprae, permanece como uma doença negligenciada e importante problema de saúde pública no Brasil(1,2). Em 2018, foram registrados aproximadamente 209 mil casos novos de hanseníase no mundo. Aproximadamente $13,0 \%$ do total desses casos ocorreram nas Américas e o Brasil foi responsável por 92,6\% dos $\operatorname{casos}^{(3)}$. No país, o coeficiente de detecção geral foi de 13,7 casos novos por 100 mil habitantes em $2018^{(4)}$. O estado do Tocantins foi o mais hiperendêmico para hanseníase no país, com um coeficiente de detecção de 84,87 casos por 100 mil habitantes ${ }^{(4)}$.

No mesmo ano, Palmas foi a capital mais hiperendêmica para hanseníase no país, com um coeficiente de detecção geral de $271,37 / 100$ mil habitantes ${ }^{(4)}$. Essa realidade epidemiológica é resultado da implementação do projeto Palmas Livre da Hanseníase (PLH), o qual teve início em março de 2016 e final em março de $2019^{(5,6)}$. Esse projeto foi desenvolvido diante da necessidade de treinamento em serviço para o alcance de indicadores que refletissem a realidade epidemiológica local, bem como para o enfrentamento da doença e redução da prevalência oculta. Nesse contexto, o fortalecimento da Atenção Primária à Saúde (APS) foi crucial e se deu tendo como estratégia prioritária a capacitação com ressignificação das práticas dos profissionais de saúde para as ações de vigilância e controle da hanseníase ${ }^{(6)}$.

Dos profissionais que compõem a equipe estratégia saúde da família, o Agente Comunitário de Saúde (ACS) desenvolve atividades de ações de controle da hanseníase no território por meio de ações educativas individuais e coletivas, nos domicílios e na comunidade. Assim sendo, a capacitação e conhecimento dos ACS sobre a hanseníase e as ações para o seu controle são fatores importantes para se avançar no enfrentamento desse importante problema de saúde pública. É fato que a efetividade da APS deve incorporar práticas desses profissionais baseadas na vigilância à saúde no território com a atuação na abordagem coletiva desse agravo, envolvendo ações educativas, busca ativa de sintomáticos dermatológicos, busca ativa de faltosos e de casos em abandono, acompanhamento de comunicantes, bem como supervisão do tratamento. Discutir sobre os fatores associados à capacitação de ACS é oportuno e necessário na medida em que vai ao encontro dos desafios atuais acerca da qualificação da Atenção Básica no foco de um controle mais efetivo da hanseníase no município de Palmas. Assim, o objetivo deste estudo foi descrever os fatores associados à capacitação de agentes comunitários de saúde para o acompanhamento de casos de hanseníase na capital do Tocantins, Brasil.

\section{METODOLOGIA}

\section{Local de estudo}

O projeto foi desenvolvido no município de Palmas, capital mais nova do país. A população estimada é de 299.127 habitantes em 2019. Este território possui uma área de $2.218,942 \mathrm{~km}^{2(7)}$. A Rede de Atenção e Vigilância em Saúde (RAVS Palmas) da
Secretaria da Saúde, instituída pela Portaria no 457/2019, estabeleceu a forma de organização do sistema municipal de saúde, que passou a ter como características definidoras o arranjo poliárquico, o trabalho em equipe, a coordenação e longitudinalidade do cuidado, a tecnologia da informação como ferramenta de trabalho, o intercâmbio e a cooperação entre os diversos pontos de atenção à saúde com o objetivo da integralidade da atenção ${ }^{(8)}$. A RAVS possui três distritos administrativos subdivididos em 8 territórios de saúde com seus respectivos Centros de Saúde da Comunidade (CSC) e demais pontos de atenção. São assim distribuídos:

I - Distrito Administrativo de Saúde da Região Norte:

a) Território de Saúde Kanela: CSC 307 Norte, CSC 403 Norte, CSC 405 Norte, CSC 409 Norte, CSC 503 Norte e CSC 603 Norte. b) Território de Saúde Apinajé: CSC 406 Norte, CSC 508 Norte, CSC Loiane Moreno e CSC 108 Sul.

II - Distrito Administrativo de Saúde da Região Central:

a) Território de Saúde Xambioá: CSC 207 Sul, CSC 403 Sul, CSC 712 Sul e CSC 806 Sul.

b) Território de Saúde Krahô: CSC Albertino Santos, CSC Sátiro Alves, CSC Valéria Martins e CSC 1304 Sul.

c) Território de Saúde Karajá: CSC Eugênio Pinheiro, CSC Aureny II, CSC Novo Horizonte, CSC Liberdade e CSC Alto Bonito.

III - Distrito Administrativo de Saúde da Região Sul:

a) Território de Saúde Javaé: CSC Bela Vista, CSC Santa Bárbara, CSC José Hermes, CSC Morada do Sol, CSC Santa Fé.

b) Território de Saúde Xerente: CSC Laurides, CSC Taquari e CSC José Lúcio.

c) Território de Saúde Pankararú: CSC Taquaruçu, CSC Mariazinha, CSC Walterly (Taquaruçu Grande) ${ }^{(8)}$. A rede de atenção conta com 85 Equipes de Saúde da Família, 75 Equipes de Saúde Bucal, 506 agentes comunitários de saúde (ACS) e 13 Núcleos Ampliados de Saúde da Família (NASF) e 1 Equipe de Consultório na Rua.

\section{Tipo de estudo e população}

Trata-se de um estudo transversal realizado em Palmas, Tocantins entre os anos de 2017 e 2018. A população de estudo foi constituída por 506 ACS vinculados à rede de atenção primária à saúde de Palmas. Desse universo, 20 estavam em desvio de função, 7 estavam nomeados para exercício de cargos, 3 estavam de licença médica e 2 de licença para interesses pessoais, portanto não fizeram parte da população de estudo. Um total de 474 ACS eram profissionais atuantes nas Equipes de Saúde da Família, sendo que, desse total, 40 estavam de férias no período da entrevista e os demais não comparecerem ao convite para a pesquisa, então foram consideradas como perdas do estudo. Fizeram parte do estudo 301 ACS (60,5\%) que trabalham na ESF do município de Palmas.

\section{Coleta de dados}

Para a coleta de dados, seguiram-se os seguintes passos: 1) apresentação do projeto ao gestor municipal; 2) apresentação do projeto aos gerentes dos centros de saúde e pactuação das datas para a realização das entrevistas junto aos ACS. Os ACS foram convidados a participar da pesquisa pelos gerentes dos Centros de Saúde da Comunidade com 10 dias de antecedência da data de entrevista. A coleta de dados foi realizada por duas pesquisadoras experientes na área, treinadas e com suporte de acadêmicos do curso de medicina para apoio e organização da logística na coleta. 
Os participantes responderam a um formulário padronizado com questões estruturadas. A coleta de dados ocorreu entre os meses de abril a setembro de 2017 e meses de agosto a outubro de 2018. Foi verificado a capacitação dos ACS em relação à hanseníase a partir do entendimento da relação da doença com as seguintes variáveis: manchas no corpo com diminuição e/ou perda da sensibilidade; manchas na pele que coçam, descamam e desaparecem sozinhas; manchas na pele com alteração de cor e sensibilidade normal; perda de pelos nas sobrancelhas $e$ cílios; corte ou queimadura sem dor nas mãos, pés, braços ou pernas; hanseníase tem cura; transmissão da hanseníase; agente causador da doença; perda da sensibilidade nas mãos/ pés, dores nos nervos, caroços pelo corpo, febre e mal-estar. As variáveis explicativas (dependentes) investigadas para este estudo foram: faixa etária, gênero, sentimento de confiança para acompanhar casos, conhecimento do modo de transmissão da hanseníase, frequência de visita domiciliar ao paciente com hanseníase, crença na necessidade de segregação de objetos de uso pessoal do paciente até o final do tratamento, casos de hanseníase na família do ACS e ACS com diagnóstico de hanseníase. A variável desfecho (independente) selecionada foi: ter realizado capacitação / treinamento / curso sobre hanseníase.

\section{Análise dos dados}

Realizou-se a análise bivariada utilizando-se o teste de quiquadrado de Pearson e o cálculo das razões de prevalência (RP) com intervalos de $95 \%$ de confiança (IC 95\%).

A variável ter realizado capacitação / treinamento / curso de formação em educação permanente sobre hanseníase (definida pela condição de o ACS ter tido algum curso de hanseníase) foi adotada como desfecho. A análise multivariada foi realizada considerando $p<0,20$ para incluir a variável no modelo logístico, e $\mathrm{p}<0,05$ para manter a variável no modelo final, porém, permaneceram no modelo as variáveis com $p>0,05$ que alteraram consideravelmente outras variáveis. As variáveis que apresentaram colinearidade não foram incluídas no modelo final. A odds ratio $(O R)$ ajustada foi usada para analisar os fatores de associação e foi considerada significativamente diferente quando o intervalo de confiança de $95 \%$ não incluiu o valor 1 .

\section{Aspectos Éticos}

O estudo foi aprovado pelo Comitê de Ética em Pesquisa. A coleta de dados foi realizada após o esclarecimento dos objetivos da pesquisa e o consentimento livre por escrito do participante.

\section{RESULTADOS}

Fizeram parte do estudo 301 ACS. A idade mínima foi de 20, e a máxima, de 67 anos. A faixa etária de maior proporção foi entre 36 a 45 anos (41,19\%), e maioria do sexo/gênero feminino (77,41\%). O território de saúde com maior concentração de ACS participantes da pesquisa foi o Xambioá (Figura 1).

Figura 1- Frequência de Agentes Comunitários de Saúde entrevistados por território de atuação profissional em Palmas, capital do Tocantins, Brasil, 2018

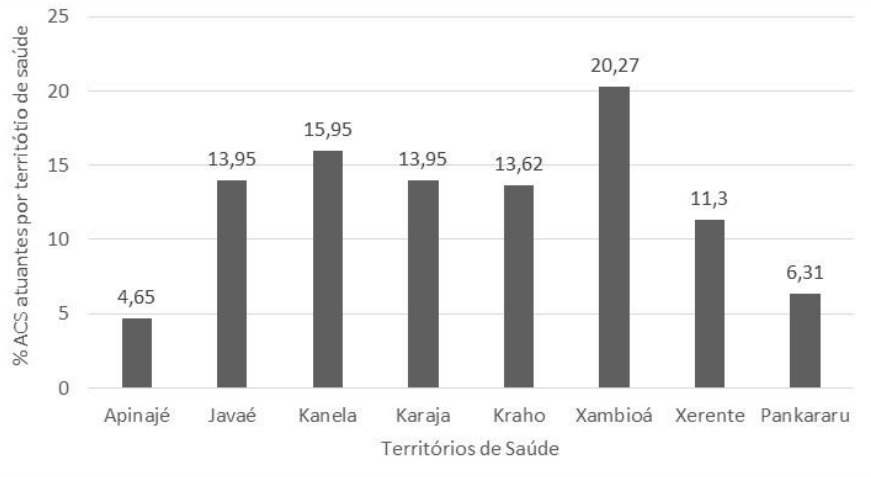

Fonte: Autores

Entre os 301 ACS entrevistados, um total de $48,84 \%$ dos ACS não reconheceu o corte ou queimadura sem dor nas mãos, pés, braços ou pernas como sinal e/ou sintoma da hanseníase. As manchas na pele com alteração de cor e sensibilidade normal foram consideradas como sinal e/ou sintoma por 30,90\% dos ACS. A perda de pelos nas sobrancelhas e cílios não foi considerada como sinal e/ou sintoma da hanseníase por $74,42 \%$ dos ACS. Mais de $50 \%$ não souberam identificar o agente causador da doença e quase $19 \%$ não souberam que a perda da sensibilidade nas mãos/ pés, dores nos nervos são sintomas da doença (Tabela 1).

Tabela 1 - Descrição dos conhecimentos dos Agentes Comunitários de Saúde sobre sinais e sintomas da hanseníase em Palmas, capital do Tocantins, Brasil, 2018

Conclusão

\begin{tabular}{|c|c|c|}
\hline Variáveis & $\mathrm{N}(301)$ & $\%$ \\
\hline \multicolumn{3}{|l|}{$\begin{array}{c}\text { Manchas no corpo com diminuição e/ou perda da } \\
\text { sensibilidade }\end{array}$} \\
\hline $\operatorname{Sim}$ & 289 & 96,01 \\
\hline Não & 12 & 3,99 \\
\hline \multirow{2}{*}{\multicolumn{3}{|c|}{$\begin{array}{c}\text { Manchas na pele que coçam, descamam e desaparecem } \\
\text { sozinhas }\end{array}$}} \\
\hline & & \\
\hline $\operatorname{Sim}$ & 28 & 9,30 \\
\hline Não & 273 & 90,70 \\
\hline \multicolumn{3}{|l|}{ Manchas na pele com alteração de cor e sensibilidade normal } \\
\hline Sim & 93 & 30,90 \\
\hline Não & 208 & 69,10 \\
\hline \multicolumn{3}{|l|}{ Perda de pelos nas sobrancelhas e cílios } \\
\hline \multicolumn{3}{|c|}{ Conc } \\
\hline Variáveis & $\mathrm{N}(301)$ & $\%$ \\
\hline Sim & 77 & 25,58 \\
\hline Não & 224 & 74,42 \\
\hline \multicolumn{3}{|l|}{$\begin{array}{c}\text { Corte ou queimadura sem dor nas mãos, pés, braços ou } \\
\text { pernas }\end{array}$} \\
\hline Sim & 154 & 51,16 \\
\hline Não & 147 & 48,84 \\
\hline \multicolumn{3}{|l|}{ A hanseníase tem cura } \\
\hline Sim & 240 & 79,73 \\
\hline Não & 14 & 4,65 \\
\hline Não soube responder & 47 & 15,61 \\
\hline \multicolumn{3}{|l|}{ Como ocorre a transmissão da hanseniase } \\
\hline $\begin{array}{c}\text { Vias aéreas (tosse e espirro) e contato íntimo e prolongado com } \\
\text { pessoas doentes }\end{array}$ & 268 & 89,04 \\
\hline $\begin{array}{l}\text { Contato com as lesões da pessoa doente em qualquer ambiente } \\
\text { independente do tempo de permanência. }\end{array}$ & 33 & 10,96 \\
\hline \multicolumn{3}{|l|}{$\begin{array}{c}\text { Via sexual, compartilhamento de seringas, objetos pessoais } \\
\text { Qual o agente causador da doença }\end{array}$} \\
\hline Bactéria & 146 & 48,50 \\
\hline Vírus & 115 & 38,21 \\
\hline Protozoário & 40 & 13.29 \\
\hline \multicolumn{3}{|l|}{$\begin{array}{l}\text { Perda da sensibilidade nas mãos/ pés, dores nos nervos, } \\
\text { caroços pelo corpo, febre e mal-estar foram reconhecidos } \\
\text { como sintomas de hanseníase }\end{array}$} \\
\hline Sim & 245 & 81,40 \\
\hline Não & 25 & 8,31 \\
\hline Não sei responder & 31 & 10,30 \\
\hline
\end{tabular}

Fonte: Autores

Os fatores associados à capacitação/treinamento de ACS para as ações de controle da hanseníase na análise bivariada são apresentados na Tabela 2. Os fatores associados à capacitação 
de ACS para o acompanhamento de casos de hanseníase foram: segrega pacientes na restrição de objetos de uso pessoal, quem maior faixa etária, mulheres, confiança para acompanhar os já teve alguém doente na família. Constatou-se escassez de casos, quem já realizou duas ou mais visitas domiciliares para estudos que abordem o tema capacitação de ACS sobre a casos de hanseníase, quem não segrega pacientes na restrição hanseníase.

de objetos de uso pessoal, quem já teve alguém doente na família, e quem já adoeceu por hanseníase.

Tabela 2- Análise bivariada de fatores associados ao conhecimento de Agentes Comunitários de Saúde sobre hanseníase no município de Palmas, capital do Tocantins, Brasil, 2018

Conclusão

\begin{tabular}{|c|c|c|c|c|c|c|}
\hline \multirow[t]{2}{*}{ Variável } & \multirow{2}{*}{$\begin{array}{c}\text { Total } \\
\mathrm{N}\end{array}$} & \multicolumn{2}{|c|}{$\begin{array}{l}\text { Realizou } \\
\text { capacitaçäo }\end{array}$} & \multirow[t]{2}{*}{$\begin{array}{l}\text { Razão de } \\
\text { Chances }\end{array}$} & \multirow[t]{2}{*}{ IC $95 \%$} & \multirow[t]{2}{*}{$\begin{array}{c}\text { Valor } \\
\text { p }\end{array}$} \\
\hline & & $\mathrm{N}$ & $\%$ & & & \\
\hline \multirow[t]{2}{*}{$\begin{array}{c}\text { Faixa etária } \\
<35 \\
36-45 \\
\end{array}$} & $\begin{array}{c}81 \\
124\end{array}$ & $\begin{array}{l}39 \\
96\end{array}$ & $\begin{array}{l}48,15 \\
77,42\end{array}$ & $\begin{array}{c}1 \\
1,55\end{array}$ & $1,20-2,00$ & 0,0000 \\
\hline & & & & & & Conclus \\
\hline \multirow[t]{2}{*}{ Variável } & Total & \multicolumn{2}{|c|}{$\begin{array}{l}\text { Realizou } \\
\text { capacitação }\end{array}$} & $\begin{array}{l}\text { Razão de } \\
\text { Chances }\end{array}$ & IC $95 \%$ & $\begin{array}{c}\text { Valor } \\
\mathbf{p}\end{array}$ \\
\hline & $\mathbf{N}$ & $\mathrm{N}$ & $\%$ & & & \\
\hline \multicolumn{7}{|l|}{ Gênero } \\
\hline Masculino & 68 & 36 & 52,94 & 1 & -5 & \multirow{2}{*}{0,0001} \\
\hline $\begin{array}{c}\text { Feminino } \\
\text { Modo de Transmissão da } \\
\text { hanseniase }\end{array}$ & 233 & 171 & 73,39 & 1,38 & $1,09-1,75$ & \\
\hline $\begin{array}{c}\text { e contato intimo e } \\
\text { prolongado com pessoas } \\
\text { doentes } \\
\text { Contato com as lesões da }\end{array}$ & 268 & 189 & 70,52 & 1 & - & 0,0061 \\
\hline $\begin{array}{c}\text { pessoa doente em qualquer } \\
\text { ambiente independente do } \\
\text { tempo de permanência. } \\
\text { Tem sentimento de medo } \\
\text { para visitar pacientes com } \\
\text { hanseniase }\end{array}$ & 33 & 18 & 54,55 & 1,29 & $0,93-1,78$ & \\
\hline Sim & 75 & 40 & 53,33 & 1 & - & \\
\hline $\begin{array}{c}\text { Não } \\
N^{\circ} \text { de VDs realizadas ao }\end{array}$ & 226 & 167 & 73,89 & 1,38 & $1,10-1,73$ & 0,0009 \\
\hline $\begin{array}{l}\text { paciente com hanseniase } \\
<4 \text { visitas domiciliar }\end{array}$ & 90 & 50 & 55,56 & 1 & - & 0,0001 \\
\hline $\begin{array}{l}\geq 4 \text { visitas domiciliares } \\
\text { Orientaçäo para separar } \\
\text { objetos de uso pessoal no } \\
\text { domicilio }\end{array}$ & 211 & 157 & 74,41 & 1,34 & $1,09-1,63$ & \\
\hline $\operatorname{Sim}$ & 69 & 29 & 42,03 & 1 & - & 0,000 \\
\hline $\begin{array}{c}\text { Não } \\
\text { Alguém na sua familia já } \\
\text { teve hanseníase }\end{array}$ & 232 & 178 & 76,72 & 1,82 & $1,37-2,42$ & \\
\hline Não & 211 & 136 & 64,45 & 1 & - & 0,0013 \\
\hline 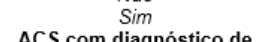 & 90 & 71 & 78,89 & 1,22 & $1,05-1,41$ & \\
\hline $\begin{array}{c}\text { ACS com diagnóstico de } \\
\text { hanseniase }\end{array}$ & & & & & & \\
\hline Não & 241 & 161 & 66,80 & 1 & - & 0,1402 \\
\hline Sim & 60 & 46 & 76,67 & 1,14 & $0,97-1,35$ & \\
\hline
\end{tabular}

Fonte: Autores

Nota: VDs- Visitas domiciliares

O resultado da regressão logística é apresentado na Tabela 3. A maior chance independente para a capacitação do ACS sobre hanseníase foi não orientar a separação de objetos de uso pessoal no domicílio (Tabela 3).

Tabela 3 - Regressão logística de fatores associados a capacitação de Agentes Comunitários de Saúde no município de Palmas, capital do Tocantins, Brasil, 2017- 2018.

\begin{tabular}{cccc}
\hline Variável & ORa ajustada & $\mathrm{LC}^{\mathrm{b}}$ & Valor $\mathrm{P}$ \\
\hline $\begin{array}{c}\text { Não orienta separação de objetos de uso } \\
\text { pessoal no domicilio }\end{array}$ & 3,42 & $1,88-6,25$ & 0,0000 \\
\hline
\end{tabular}

Fonte: Autores

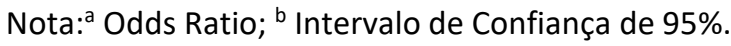

\section{DISCUSSÃO}

Os resultados desse estudo mostram evidências de que os fatores associados à maior capacitação dos ACS para acompanharem os casos de hanseníase no território de abrangência foram: maior faixa etária, mulheres, confiança para acompanhar os casos, quem já realizou duas ou mais visitas domiciliares para casos de hanseníase, quem não

Por outro lado, as lacunas de conhecimento dos ACS sobre os sinais, sintomas e modo de transmissão da doença apontaram a necessidade de educação permanente bem como a implementação de projetos de intervenção similares ao projeto Palmas Livre da Hanseníase na formação de ACS, o qual comprovou a efetividade e potencialidade do treinamento proposto para as ações de diagnóstico e controle da hanseníase no município de Palmas. O estudo resultante do projeto Palmas Livre da Hanseníase trouxe ainda, evidências de que a agilidade diagnóstica dos serviços de atenção primária resulta em indicadores que refletem a incidência real de ocorrência de casos, bem como na redução da prevalência oculta em áreas hiperendêmicas, a qual é a maior responsável pela transmissão da doença ${ }^{(6)}$.

Essas lacunas de conhecimento podem prejudicar a busca ativa de sintomáticos dermatológicos e de contatos de casos, pois o papel do ACS é essencial para a interrupção da cadeia de transmissão da hanseníase à medida, principalmente, que estes reconhecem os sintomas da doença, encaminham corretamente os casos suspeitos para investigação do diagnóstico e colaboram para o início precoce do tratamento ${ }^{(9)}$. Há relatos na literatura com resultados e conclusões semelhantes a este estudo, em que ACS também apresentaram lacunas no conhecimento sobre esse tema ${ }^{(10)}$. Em um outro estudo, o conhecimento dos ACS sobre a hanseníase mostrouse insuficiente, situação em que $9,8 \%$ dos ACS desconhecia o fato de a hanseníase ser contagiosa, $47,6 \%$ não sabia a sua etiologia, 1,9\% a consideravam uma doença não tratável e 3,3\% ignoravam quais seriam os seus sintomas ${ }^{(11)}$. Por outro lado, em mais outro estudo, foi apontado que os ACS consideram sua formação profissional insuficiente e as principais falhas percebidas foram: excesso de padronização de conteúdos que abordam temas predominantemente técnico-científicos e que não incluem dados da realidade local; enfoque insuficiente em aspectos teóricos e práticos que poderiam auxiliá-los no enfrentamento de questões da realidade cotidiana de trabalho, como o manejo de problemas familiares e de ordem social ${ }^{(12)}$.

É fato que as qualificações se dão, muitas vezes, por meio de capacitações ou de atualizações quase sempre pontuais, o que pode se refletir como uma fragilidade por não proporcionarem uma abordagem contínua dos problemas encontrados na prática profissional, o que pode limitar transformações dessas práticas $^{(9)}$. De fato, as capacitações em modelos tradicionais parecem ser insuficientes, refletindo a fragilidade da educação em saúde, que, consequentemente, provoca poucas mudanças e resultados nos cenários ${ }^{(6)}$.

Em estudo recente realizado no mesmo cenário, foi apontado que a execução do treinamento para hanseníase na atenção primária valoriza a capacidade operacional do serviço, nos aspectos de observação, análise, reflexão, questionamento e busca de soluções e respostas de modo participativo em face da falta de habilidade e insegurança para as ações de controle da hanseníase, e isso pode ser referenciado para os $\operatorname{ACS}^{(6)}$. É fato que um bom nível de conhecimento dos ACS sobre o diagnóstico, transmissão e tratamento da hanseníase promove 
dispersão de informações úteis aos usuários para quebra da cadeia de transmissão e redução de novos $\operatorname{casos}^{(13,14)}$.

Há ainda que se pensar que, na ação dos ACS, há uma interrelação de saberes, construídos local e exteriormente, e é em suas práticas cotidianas que eles realizam uma negociação entre os saberes e produzem suas próprias ações baseadas em sua forma de ver o mundo(15). Adicionalmente, os ACS mais velhos foram significativamente mais capacitados e isso pode ser devido a estes profissionais trazerem consigo conhecimentos e experiências vivenciados em um território hiperendêmico para hanseníase. Há uma pesquisa que aponta que ACS mais velhos tendem a ter seus conceitos sobre o processo saúde doença melhor definidos. Em contraponto, para os ACS mais jovens, os conceitos e definições de saúde e doença parecem não estar tão elucidados e enraizados ${ }^{(16)}$.

Quanto à abordagem por gênero, as ACS do sexo feminino apresentaram maior razão de chances quanto à capacitação para acompanhar casos de hanseníase, possivelmente é um fato decorrente das atividades inerentes à profissão, uma vez que as mulheres, geralmente, possuem maior sensibilidade para cuidar, orientar, zelar pelo bem-estar físico e social de toda família e se responsabilizar pelos dilemas da comunidade $^{(17)}$. O predomínio feminino reforça o estereótipo da mulher como figura responsável pelo cuidado. Este é um ponto importante que também justifica esse resultado, pois a função do ACS possibilita a inserção ou reinserção no mercado de trabalho e o incremento da renda familiar sem necessidade de se afastar da comunidade onde mora ${ }^{(18,19)}$. Na sociedade patriarcal brasileira a mulher, normalmente, é responsável pela realização/gerenciamento das atividades domésticas, educação e cuidados com os filhos além de prestar atenção aos membros idosos da família. É importante ressaltar que essa diferença ocasionada entre o sexo feminino e masculino pode ser devido à maior dificuldade dos ACS do sexo masculino exercerem a sua função, pois há um constrangimento das mulheres em recebê-los em casa quando estão sozinhas e também de conversar sobre assuntos específicos, como orientações acerca de exames ginecológicos e câncer de mama, o que dificulta o estabelecimento do vínculo necessário para a realização de um trabalho efetivo ${ }^{(20)}$.

Os resultados também demonstraram que a capacitação de ACS em hanseníase foi estatisticamente significativa para aqueles que realizaram quatro ou mais visitas domiciliares a pessoas acometidas por hanseníase. É esperado que a experiência profissional no acompanhamento de casos promova maior conhecimento ou capacidade no assunto. Abordagem semelhante foi realizada em uma pesquisa realizada em Belo Horizonte com ACS sobre o conhecimento de tuberculose (TB), e mostrou maior conhecimento entre aqueles indivíduos que acompanhavam um maior número de famílias com TB, mas não foi estatisticamente significante a diferença como encontrada em nosso estudo ${ }^{(21)}$.

O fato de os ACS não considerarem necessário a segregação de pessoas com hanseníase na restrição de objetos de uso pessoal foi estatisticamente significante para aqueles que se consideraram capacitados e foi o único fator determinante na análise multivariada. Esse achado corrobora com os resultados de estudos realizados com profissionais de atenção primária à saúde em Taiwan e no Brasil, em que o treinamento em serviço para atuação no campo não apenas promove o conhecimento, mas também reduz o estigma e melhora as ações de controle para doenças negligenciadas como a hanseníase e a $\mathrm{TB}^{(6,22)}$. Ademais, a execução de treinamento profissional nas unidades de atenção primária valoriza a capacidade operacional do serviço e a busca de soluções e respostas de modo participativo em face da falta de habilidade e insegurança para a execução das ações de controle da hanseníase ${ }^{(6)}$.

Houve diferença estatística para maior capacitação, os ACS que relataram a presença de um familiar com hanseníase e ainda a condição de o ACS ter a doença. Isso pode ser explicado devido as memórias que guardam do tratamento, como também obtêm a oportunidade de acessar outros saberes, técnicos e científicos, sobre a hanseníase, suas manifestações, tratamento, cuidados e seus desfechos. Há relatos na literatura que mulheres que foram diagnosticadas com hanseníase passam a exercer o papel de difusoras do conhecimento científico, na medida em que, ao aderirem ao tratamento e participarem dos programas de educação em saúde, adquirem informações e as repassam para a sociedade (família, amigos, entre outros), contribuindo para a inserção de outros elementos que irão se congregar na desconstrução e reconstrução de representações sociais da hanseníase ${ }^{(23)}$.

As limitações desse estudo podem ser apontadas considerando o fato de o estudo ter sido conduzido utilizando-se um instrumento não validado para avaliar a capacitação de ACS sobre hanseníase. Para adequação, métodos de análise estatística como a Teoria da Resposta ao Item (TRI) podem ser utilizados com o intuito de validação e efeito de comparabilidade dos dados ${ }^{(24)}$. Adicionalmente, o questionário aplicado para a medida de resultado pediu informações a respeito de práticas profissionais, assim, pode ter havido viés de memória ou dicotomia nas respostas entre o que é desempenhado na prática profissional e o que é considerado socialmente aceitável.

\section{CONCLUSÃo}

Por fim, este estudo apontou a necessidade de educação permanente para a reorientação das práticas profissionais de ACS, pois os resultados trouxeram evidências das lacunas de conhecimento sobre a doença e os fatores associados à capacitação. O controle da hanseníase será possível quando as ações realizadas na esfera da Estratégia Saúde da Família forem focadas considerando a formação da equipe multiprofissional com ênfase no ACS, pois este profissional é o responsável pela busca ativa de casos suspeitos no território. Os resultados apresentaram novas informações na literatura científica sobre capacitação dos ACS acerca da hanseníase e poderá subsidiar estratégias destinadas ao aperfeiçoamento das atividades de controle da doença.

\section{REFERÊNCIAS BIBLIOGRÁFICAS}

1. 1. Lockwood DN, Shetty V, Penna GO. Hazards of setting targets to eliminate disease: lessons from the leprosy elimination campaign. BMJ 2014; 348:g1136. DOI: 10.1136/bmj.g1136. 
2. 2. Lockwood DN, Suneetha S. Leprosy: too complex a disease for a simple elimination paradigm. Bull World Health Organ 2005; 83:230-5 [Cited 2020 Mar 5]. Available from: https://pubmed.ncbi.nlm.nih.gov/15798849/.

3. 3. World Health Organization. Global leprosy update, 2017: reducing the disease burden due to leprosy. Wkly Epidemiol Rec 2018; 93; 445-456. ORGANIZATION, W. H. South-East Asia 2016 [Cited 2020 Mar 2]. Available from: https://apps.who.int/iris/bitstream/handle/10665/27429 0/WER9335-445-456. pdf?sequence=1\&isAllowed=y.

4. 4. Brasil. Ministério da Saúde, Secretaria de Vigilância em Saúde. Boletim Epidemiológico de Hanseníase - 2020. Bol Epidemiol. 2020 [Cited 2020 Mar 5]. Available from: https://portalarquivos2.saude.gov.br/images/pdf/2020/i aneiro/31/Boletim-hanseniase-2020-web.pdf.

5. 5. Palmas. Secretaria Municipal de Saúde. Portaria no 518, de 14 de junho de 2016. Diário Oficial do Município de Palmas 2016; 14 jun [Cited 2020 Apr 14]. Available from:

https://www.jusbrasil.com.br/diarios/119243720/dompmw-normal-28-06-2016-pg-12.

6. 6. Monteiro LD, Lopes LSO, Santos PR dos, Rodrigues ALM, Bastos WM, Barreto JA. Tendências da hanseníase após implementação de um projeto de intervenção em uma capital da Região Norte do Brasil, 2002-2016. Cad. Saúde Pública 2018; 34 (11). DOI: 10.1590/0102$311 \times 00007818$

7. 7. Instituto Brasileiro de Geografia e Estatística (IBGE). [homepage na internet]. Cidades e Estados. Panorama. População. Território e ambiente [Cited 2020 Mar 6]. Available from: https://cidades.ibge.gov.br/brasil/to/palmas/panorama.

8. 8. Secretaria Municipal de Saúde. Portaria № 457/2019, de 15 de abril de 2019. Diário Oficial do Município de Palmas 2019; 15 abr [Cited 2020 Mar 2]. Available from: https://www.jusbrasil.com.br/diarios/237444649/dompmw-normal-15-04-2019-pg-8.

9. 9. Oliveira CM de, Linhares MSC, Neto FRGX, Mendes IMVP, Kerr LRFS. Conhecimento e práticas dos Agentes Comunitários de Saúde sobre hanseníase em um município hiperendêmico. Saúde Rev. 2018;18(48):39-50 [Cited 2020 Mar 2]. Available from: https://www.metodista.br/revistas/revistasunimep/index.php/sr/article/viewFile/3338/2182.

10. 10. Andrade CG de, Costa ICP, Freire MEM, Santos KFO dos, Gouveia EM de L, Claudino HG e. Hanseníase: Compreensão De Agentes Comunitários De Saúde. Rev Bras Ciências da Saúde. 2011;15(1):17-24. DOI:10.4034/RBCS.2011.15.01.03

11. 11. Filho MM, Gomes CFL. Preconceito e conhecimento sobre hanseníase: a situação do agente comunitário de saúde. Rev Bioethikos. 2014;8(2):153-160 [Cited 2020 Apr 23]. Available from: https://saocamilosp.br/assets/artigo/bioethikos/155563/A03.pdf.

12. 12. Alonso CMC, Béguin PD, Duarte FJCM. Trabalho dos agentes comunitários de saúde na Estratégia Saúde da
Família: metassíntese. Rev Saúde Pública. 2018; 52:14. DOI: 10.11606/S1518-8787.2018052000395.

13. 13. Ribeiro FS, Silva MLA, Mendonça ALB, Soares JSA, Freitas CSL, Linhares MSC. Qualidades dos serviços prestados pelos centros de saúde da família de Sobral Ceará aos portadores de hanseníase nos anos de 20092010. Sanare (Sobral). 2012;11(2):44-51 [Cited $2020 \mathrm{Mar}$ 5]. Available from: https://sanare.emnuvens.com.br/sanare/article/view/27 $\underline{5}$.

14. 14. Ximenes FRG Neto, Martins FR, Liberato BTG, Carvalho JP Filho, Aguiar BEM, Martins AR. Ações de sustentabilidade para o controle da hanseníase: a experiência do município Cariré - Ceará. Sanare (Sobral). 2011;10(2):71-4 [Cited 2020 Mar 5]. Available from: https://sanare.emnuvens.com.br/sanare/article/viewFile 2258/231.

15. 15. Lotta GS. Saberes locais, mediação e cidadania: o caso dos agentes comunitários de saúde. Saúde soc. 2012;21(Suppl 1):210-222. DOI: 10.1590/S010412902012000500018.

16. 16. Santana TB, Azevedo BDS, Maia ACD.-S A. Fatores associados ao conhecimento de agentes comunitários de saúde em relação à saúde bucal. Cad. Saúde Colettiva.2018; 26(3): 292-297. DOI: 10.1590/1414$\underline{462 \times 201800030420}$

17. 17. Moura MS de, Carvalho CJ de, Amorim JTC de, Marques MFSS, Moura L de FA de D, Mendes RF. Perfil e práticas de saúde bucal do agente comunitário de saúde em municípios piauienses de pequeno porte. Ciênc. Saúde coletiva. 2010;15(Suppl 1):1487-1495. DOI: 10.1590/S1413-81232010000700061.

18. 18. Barbosa RHS, Menezes CAFM, David HMSL, Bornstein VJ. Gênero e trabalho em saúde: um olhar crítico sobre o trabalho de agentes comunitárias/os de Saúde. Interface - Comunic Saude Educ. 2012;16(42):751-65. DOI: 10.1590/S1414-32832012000300013.

19. 19. Nogueira RP, Silva FB, Ramos ZVO. A vinculação institucional de um trabalhador sui generis: o agente comunitário de saúde. Rio de Janeiro: Instituto de Pesquisa Econômica Aplicada; 2000. (Texto para Discussão; $n^{\circ}$ 735) [Cited 2020 Apr 11]. Available from: https://www.ipea.gov.br/portal/index.php?option=com content \&view=article\&id $=4208$.

20. 20. Wai MFP. O trabalho do agente comunitário de saúde na estratégia de saúde da família: fatores de sobrecarga e mecanismo de enfrentamento [dissertação]. Ribeirão Preto: Universidade de São Paulo; 2007. DOI: 10.11606/D.22.2007.tde-08082007-104445.

21. 21. Rocha GSS, Lima MG, Moreira JL, Ribeiro KC, Ceccato $\mathrm{M}$ das GB, Carvalho $\mathrm{W}$ da $\mathrm{S}$ et al. Conhecimento dos agentes comunitários de saúde sobre a tuberculose, suas medidas de controle e tratamento diretamente observado. Cad. Saúde Pública. 2015;31(7):1483-1496. DOI: 10.1590/0102-311X00112414.

22. 22. Wu PS, Chou P. Characteristics of directly observed treatment short-course workers in Taiwan. J Chin Med 
Assoc. 2013;76(1):48-52. DOI:

10.1016/j.jcma.2012.08.014

23. 23. Palmeira IP, Ferreira $M$ de A. "O corpo que eu fui e o corpo que eu sou": concepções de mulheres com alterações causadas pela hanseníase. Texto contexto enferm. Florianópolis, v. 21, n. 2, p. 379-386, June 2012 . DOI: $10.1590 / \mathrm{S} 0104-07072012000200016$.

24. 24. Araujo EAC de, Andrade DF de, Bortolotti SLV. Teoria da Resposta ao Item. Rev Esc Enferm USP. 2009;

43(Esp):1000-8. DOI: 10.1590/S0080-

$\underline{62342009000500003}$ 